\title{
Culprit Medications and Risk Factors Associated with Stevens-Johnson Syndrome and Toxic Epidermal Necrolysis: Population-Based Nested Case-Control Study
}

\author{
Naomi Gronich ${ }^{1,2}\left(\mathbb{D} \cdot\right.$ David Maman $^{1} \cdot$ Nili Stein $^{2} \cdot$ Walid Saliba $^{1,2}$ \\ Accepted: 21 November 2021 / Published online: 4 February 2022 \\ (c) The Author(s), under exclusive licence to Springer Nature Switzerland AG 2022
}

\begin{abstract}
Objective Our objective was to describe the incidence of Stevens-Johnson syndrome/toxic epidermal necrolysis (SJS/TEN) in a large unselected cohort, to validate the culprit drugs involved and the frequency of SJS/TEN for each drug, and to analyze the clinical risk factors for SJS/TEN.

Methods Using the computerized database of Clalit Health Services, we identified all adult patients with a new SJS/TEN diagnosis between 1 January, 2008 and 30 June, 2019 and validated each case. Cumulative incidence of SJS/TEN for each culprit drug was calculated by dividing the number of valid cases by the total number of new users of the drug in the study period. Using risk-set sampling, 20 controls were matched to each case by sex and age on the index date for a nested casecontrol analysis. Multivariable conditional logistic regression was used to estimate the odds ratio and $95 \%$ confidence interval for the association of incident SJS/TEN with chronic diseases.

Results We identified 87 adult cases of true/probable SJS/TEN between 1 January, 2008 and 30 June, 2019. Culprit drugs [with ALDEN scores ascertained as at least probable $(\geq 4)$ ] associated with the highest absolute risks were phenytoin, lamotrigine, and allopurinol with 3.56, 2.82, and 1.10 SJS/TEN cases/10,000 new users, respectively. Additional drugs with mean ALDEN scores $\geq 4$ were sunitinib, sulfasalazine, carbamazepine, etoricoxib, etodolac, and cefuroxime, cumulative incidence: $13.57,0.72,0.32,0.05,0.02$, and 0.02/10,000 new users, respectively. Previous diagnosis of systemic lupus erythematosus, psoriasis, previous drug allergies, epilepsy, malignancy, history of cerebrovascular accident, and history of diabetes mellitus were associated with an increased risk for SJS/TEN, odds ratios (95\% confidence interval):17.41 (1.31-230.72), 10.28 (3.61-29.31), 5.21 (2.95-9.19), 4.92 (1.88-12.85), 3.17 (1.77-5.66), 2.61 (1.26-5.41), and 1.98 (1.12-3.53), respectively. Conclusions Attention should be drawn to drugs assessed by high ALDEN scores that were associated with high absolute risks for SJS/TEN. Psoriasis, former drug allergies, in addition to systemic lupus erythematosus, malignancy, history of cerebrovascular accident, and diabetes mellitus were associated with increased SJS/TEN risk in our analysis.
\end{abstract}

\section{Introduction}

Stevens-Johnson syndrome (SJS) and toxic epidermal necrolysis (TEN) are rare mucocutaneous adverse drug reactions, with estimated incidence rates from 1 to 12 per million person-years, and reported mortality ranges of $1-9 \%$ for SJS and 30-50\% for TEN [1-4]. Epidermal necrolysis results in

Naomi Gronich

gronichn@clalit.org.il

1 Ruth and Bruce Rappaport Faculty of Medicine, TechnionIsrael Institute of Technology, Haifa, Israel

2 Department of Community Medicine and Epidemiology, Lady Davis Carmel Medical Center, 7 Michal St., 34362 Haifa, Israel extensive blisters and detachment of skin and mucous membranes, with severe early and late complications in surviving patients. Stevens-Johnson syndrome and TEN are two forms of epidermal necrolysis, differing only with respect to the amount of skin detachment relative to the body surface area involved [1]. The prevalent pathophysiology concept is that cytotoxic $\mathrm{T}$ lymphocytes play a role in the initiation phase of SJS/TEN [2, 5-7].

The risk of SJS/TEN seems to be limited to the first 8 weeks of treatment. Various anti-epileptic drugs, sulfonamide antibiotics, allopurinol, antiretroviral drugs, and oxicam analgesics have repeatedly been identified as key triggers of SJS/TEN [1]. Reports of potential associations between SJS/TEN and a variety of other drugs are abundant, albeit based on little evidence, such as for sildenafil, duloxetine, glimepiride, and more. Official drug product labels 


\section{Key Points}

Medical records were used to validate the diagnosis and culprit drug/s of each Stevens-Johnson syndrome/toxic epidermal necrolysis case that occurred, in a communitybased unselected cohort of 4.7 million patients between 2008 and 2019.

Cumulative incidence of Stevens-Johnson syndrome/ toxic epidermal necrolysis was calculated for each drug, based on the total number of new users in the community.

Novel risk factors identified in association with StevensJohnson syndrome/toxic epidermal necrolysis were psoriasis, former drug allergies, history of cerebrovascular accident, and history of diabetes mellitus, in addition to known associations with systemic lupus erythematosus and malignancy. These associations require confirmation in other studies.

and national formularies list SJS/TEN as a potential adverse reaction to these drugs though not established on firm data [8-10].

Identification of the drug responsible for the disease (culprit drug) is crucial for regulatory and also for clinical decisions. In the acute phase, withdrawal of the suspected medication is an urgent requirement in order to improve the immediate prognosis, while other treatments often need to be continued. Avoiding re-exposure to the culprit drug is crucial, after the patient has recovered, while permitting the use of other medicines [11]. The algorithm of drug causality for EN (ALDEN) [11] is useful in order to improve the individual assessment of drug causality.

For allopurinol, carbamazepine, and dapsone, an association was found between specific HLA alleles and the risks for SJS/TEN $[12,13]$. Data on associated diseases are scarce. Systemic lupus erythematosus, human immunodeficiency virus, malignancy, and status post-bone marrow transplant were described as associated with SJS/TEN from small data sets. An aberrant T-cell function might be a common denominator in these high-risk situations [5].

Epidemiologic data on SJS/TEN are limited. Previous studies have focused primarily on identifying drugs that cause SJS/TEN using hospital based case-control studies; risk estimation was probably inaccurate. Large electronic databases are an important tool in epidemiologic research and can be particularly useful in conducting populationbased studies on rare outcomes. Still, there is paucity of studies on SJS/TEN using these data sources $[4,14]$. We sought to determine the incidence of SJS/TEN in a large population database in Israel, to assess culprit drugs and the cumulative incidence of SJS/TEN with each culprit drug, and to describe clinical characteristics of the affected patients and the associated risk.

\section{Patients and Methods}

The study is based on the computerized database of Clalit Health Services, which provides inclusive healthcare for 4.7 million patients (53\% of the population). Healthcare coverage in Israel is mandatory according to the National Health Insurance Law (1995), and is provided by four groups akin to not-for-profit health maintenance organizations. All members of the different health maintenance organizations have similar health insurance plans and similar access to health services, including low medication copayments. The electronic medical records of Clalit include data from multiple sources: records of primary care physicians, community specialty clinics, hospitalizations, laboratories, and pharmacies. Data on drugs include all outpatient prescriptions as well as drug dispensing in pharmacies. Because of the low copayment, Clalit patients buy drugs in Clalit pharmacies (or in general pharmacies that report to Clalit), thus drug dispensing data give a good representation of medications acquired. Clalit pools data from its many operational systems into a unified analytic data warehouse. A registry of chronic disease diagnoses is compiled from these data sources. Diagnoses are captured in the registry by diagnosis-specific algorithms, employing International Classification of Diseases, Ninth Revision (ICD-9) code reading, computerized text recognition of diagnoses without an ICD-9 code, laboratory test results, and disease-specific drug usage. A record is kept of the data sources and dates used to establish the diagnosis, with the earliest recorded date, from any source, considered to be the defining date of diagnosis. High-quality population-based studies have been conducted based on data retrieved from the Clalit database $[15,16]$. The Lady Davis Carmel Review Board approved the study protocol.

We identified all adult patients in Clalit by the ICD-9 codes for SJS/TEN $(695.13,695.14,695.15)$, or by computerized text recognition of diagnoses without an ICD-9 code, using the keywords "Stevens Johnson," "Stevens-Johnson," or "toxic epidermal," received between 1 January, 2008 and 30 June, 2019. The year 2008 was the beginning of our study period because until 2008 there was no distinction in ICD-9 codes between SJS/TEN and the milder erythema multiforme disease. The new SJS/TEN-specific ICD-9 codes were introduced in Israel around mid-2008.

Two researchers read the discharge/mortality letters of the patients identified. If the discharge letter from SJS/TEN hospitalization was not found, we looked for outpatient clinic visit summaries describing the hospitalization. Because SJS/ 
TEN is a severe state that cannot be managed in the outpatient setting, a patient with no report of hospitalization with a SJS/TEN discharge diagnosis was regarded as an invalid case.

From available discharge letters or outpatient visits describing the hospitalizations, we first confirmed a true new SJS/TEN diagnosis. If a diagnosis was not certain but was nevertheless the physicians working diagnosis, we regarded it as a probable diagnosis. All cases validated as true/probable SJS/TEN cases comprised this study cohort. Second, we read the medical team assessment of culprit drug/s, to be used as the source for the cases' culprit drugs. Third, we collected data needed to calculate the ALDEN score for the strength of causality, and fourth, we collected data needed to calculate the SCORTEN score for the severity assessment [17].

The ALDEN score for causality assessment of SJS/TEN (range: -12 to 10) is based on six parameters [11]: time lag from initiation of drug intake to onset of reaction; probability that the drug was present in the body at the onset of reaction; pre-challenge (administration of the same/similar drug [up to the fourth Anatomic Therapeutic Chemical Classification System level]) to the same patient in the past/rechallenge; dechallenge (drug that had been continued beyond the progression phase of the disease); drug notoriety; and other possible etiologic alternatives. For the drugs' "notoriety" parameter, we used the published table [18].

Discharge letters had the information to calculate the ALDEN score for most of the cases. Data were missing for the calculation of ALDEN scores for seven possible drugs (as shown in Table S1 of the Electronic Supplementary Material [ESM]).

SCORTEN score (range: 0-7) is a severity-of-illness score developed for cases of TEN that included seven variables: age $>40$ years, malignancy, tachycardia $>120$ / min, initial percentage of epidermal detachment $>10 \%$ of body surface area, serum urea $>10 \mathrm{mmol} / \mathrm{L}$, serum glucose $>14 \mathrm{mmol} / \mathrm{L}$, and bicarbonate $<20 \mathrm{mmol} / \mathrm{L}$ [17]. For two patients that died of TEN, there were no data for the SCORTEN score calculation (Table S1 of the ESM). In addition, in a few patients, there were missing values as follows: bicarbonate laboratory test was not performed in 12 cases, and we used the zero points in these cases based on the clinical evaluation of the cases, and the assumption that the blood $\mathrm{pH}$ test had not been performed in these cases because of the mildness of disease. Similarly, descriptions of body surface areas involved were missing for five mild cases; for nine patients, heart rate measurements were missing in the discharge letters; for two patients, serum glucose and serum urea levels were unavailable. We used the zero points for these parameters as well, using similar clinical judgments of the low severity of these clinical cases.
The incidence of SJS/TEN/year was calculated by dividing the number of validated adult cases diagnosed each year by the number of adult insurees in the midst of the year. Cumulative incidence of SJS/TEN for each culprit drug was calculated by the total number of valid adult cases associated with the drug divided by the number of Clalit adult new users of the drug between 1 January, 2008 and 30 June, 2019. A new user was defined as an insuree who have been dispensed at least one prescription of the drug, for the first time since 2002 (when full electronic data were available in Clalit).

We present descriptive statistics using counts and percentages for categorical variables, and means and standard deviations for continuous variables. To identify risk factors for SJS/TEN, we performed a nested case-control analysis. The date of SJS/TEN was defined as the index date for the cases and controls. For each case, 20 controls were randomly selected from Clalit using risk set sampling. Controls had to be alive at the index date and have the same sex and same age \pm 1 year at the index date. For each case and control, we extracted demographics and chronic disease diagnoses, only if the patient had received the diagnosis up to 1 day before SJS/TEN hospitalization, or the index date for controls. The ICD-9 codes used for diagnoses of former allergic reactions were V14, V14.x, and V15.08 and were extracted from the electronic health records (EHRs), similarly, if received before index dates. It should be noted that recording allergies by the primary care physician is mandatory in Israel. We used a similar procedure of data extraction for chronic diseases and former allergies for cases and controls (and not information from the discharge letters extracted manually), to prevent the creation of a differential information bias.

Conditional logistic regression was used to estimate the odds ratio (OR) with $95 \%$ confidence intervals (CIs), for an incident SJS/TEN event associated with chronic diseases known before the SJS/TEN date, relative to no disease. A multivariate analysis was performed following a forwardselection application. A $p$ value $<0.05$ was considered statistically significant. Data analysis was performed using SPSS Statistics version 24 (IBM, New York, NY, USA) and SAS version 9.4 (SAS Institute Inc., Cary, NC, USA).

\section{Results}

We identified 173 adult patients in Clalit by the ICD-9 codes (146 patients) or the words "Stevens Johnson" or "toxic epidermal" appearing in their diagnoses lists (additional 27 patients) between 1 January, 2008 and 30 June, 2019. Following interrogation in the EHRs, there were 68 patients with a true valid diagnosis, and 19 patients with a probable SJS/TEN diagnosis (to a total of 87 cases of true/ probable SJS/TEN). An additional ten patients with SJS/ 
TEN occurred before 2008; an additional 70 patients had final non-SJS/TEN diagnoses (most of them had been finally diagnosed with erythema multiforme, or other acute drug eruption or had acute or chronic skin disease); and an additional six patients' EHRs were not helpful, either because the patients were not insured by Clalit on the day of validation and the medical file could not be reached or because SJS/ TEN discharge letters or outpatient visits were absent from the patients' EHRs.

The incidence of SJS/TEN within Clalit adult insurees in Israel by years is shown in Table 1. Of the cohort of 87 patients, 55 were female (63\%). Mean age was 56.96 years (range 20-88 years). Thirty-three (37.9\%) were smokers (ever).

We summarized all potential drugs involved in the 82 true/probable SJS/TEN cases in whom culprit drug/s were identified. There were 38 distinct drugs, listed in Table 2 and presented in Fig. 1. Culprit drugs [with ALDEN scores ascertained as at least probable $(\geq 4)]$ that were associated with the highest absolute risks were the antiepileptic medications phenytoin and lamotrigine and the anti-gout medication allopurinol with 3.56, 2.82, and 1.10 SJS/TEN cases/10,000 new users in the community, respectively (Table 2; Fig. 1). The tyrosine-kinase inhibitor sunitinib also had an ALDEN score of 4 and a high cumulative incidence (13.57/10,000 new users) but only one case was reported.

Out of 11 patients with SJS/TEN attributed to allopurinol: the first had received allopurinol with antineoplastic treatment for multiple myeloma, and a second patient had been recently diagnosed with endometrial adenocarcinoma and was prescribed allopurinol by the nephrologist to prepare for chemotherapy treatment. The other nine patients had not been diagnosed with malignant disease before the SJS/TEN diagnosis.

Other drugs with mean ALDEN scores $(\geq 4)$ were sulfasalazine, the antiepileptic carbamazepine, COX-2 inhibitors etoricoxib and etodolac, and the cephalosporin cefuroxime, with a cumulative incidence of $0.72,0.32,0.05,0.02$, and 0.02 for 10,000 new users, respectively.

Drugs for which we found a possible association (ALDEN scores: 2-3) were the antiepileptic drugs valproic acid and topiramate, the anti-infectious sulfonamide trimethoprimsulfamethoxazole, clindamycin, cabozantinib, levomepromazine, and hydroxychloroquine with a cumulative incidence of more than 1 case/100,000 new users. In addition, the penicillins amoxicillin and amoxicillin-clavulanate, the quinolone ciprofloxacin, the cephalosporins cefazolin and ceftriaxone, the macrolide antibiotic roxithromycin, the antiviral acyclovir, and finally, the antifungal terbinafine with a low cumulative incidence (Table 2; Fig. 1).

Pralatrexate, carfilzomib, and bendamustine had a low probability of association by the ALDEN score, but a high
Table 1 Incidence rate of SJS/TEN in Clalit, 2008-19

\begin{tabular}{llll}
\hline Year & $\begin{array}{l}\text { Number of adult } \\
\text { insurees }\end{array}$ & $\begin{array}{l}\text { Number of SJS/ } \\
\text { TEN cases }\end{array}$ & $\begin{array}{l}\text { Incidence/million } \\
\text { adult insurees/year }\end{array}$ \\
\hline $2008^{\mathrm{a}}$ & $2,660,606$ & 2 & 1.5 \\
2009 & $2,689,199$ & 4 & 1.49 \\
2010 & $2,716,779$ & 5 & 1.84 \\
2011 & $2,753,335$ & 9 & 3.27 \\
2012 & $2,792,502$ & 5 & 1.79 \\
2013 & $2,837,081$ & 10 & 3.52 \\
2014 & $2,880,613$ & 9 & 3.12 \\
2015 & $2,922,966$ & 6 & 2.05 \\
2016 & $2,945,051$ & 10 & 3.40 \\
2017 & $2,985,056$ & 15 & 5.03 \\
2018 & $3,024,319$ & 8 & 2.65 \\
$2019^{\mathrm{b}}$ & $3,059,850$ & 4 & 2.61 \\
& & 87 & \\
\hline
\end{tabular}

ICD-9 International Classification of Diseases, Ninth Revision, SJS Stevens-Johnson syndrome, TEN toxic epidermal necrolysis

${ }^{\text {a}} \mathrm{SJS} / \mathrm{TEN}$-specific ICD-9 codes were newly introduced around mid2008 , thus the incidence rate/ 6 months is doubled to account for the incidence rate/year

${ }^{b}$ End of study: 29.6 .19 , thus the incidence rate/6 months is doubled to account for the incidence rate/year

cumulative incidence (Table 2; Fig. 1). A list of all cases can be found in Table $\mathrm{S} 1$ of the ESM.

We performed a nested case-control analysis to evaluate risk factors associated with SJS/TEN. Following application of a forward-selection algorithm, seven variables were retained in the multivariable model (Table 3). We identified novel associations with a previous diagnosis of psoriasis, adjusted OR (95\% CI) 10.28 (3.61-29.31), and with a previous allergy to any medicine with adjusted OR (95\% CI) 5.21 (2.95-9.19). We also confirmed previously described associations with systemic lupus erythematosus and malignancy, adjusted ORs of 17.41 (1.31-230.72), 3.17 (1.77-5.66), respectively. We also noted risks associated with epilepsy, former diagnosis of cerebrovascular accident, and diabetes mellitus, ORs 4.92 (1.88-12.85), 2.61 (1.26-5.41), and 1.98 (1.12-3.53), respectively (Table 3 ).

There were six fatal cases within 87 true/probable SJS/ TEN cases (case fatality of $6.9 \%$ ). Using four available SCORTEN scores for the fatal cases (because of missing discharge letters for two fatal cases), the mean SCORTEN score for the fatal cases was 3.75 (standard deviation [SD] 1.09). The mean SCORTEN score for the non-fatal cases was 1.80 (SD 1.16). Four of the six fatal cases had malignant diseases and one had also undergone an allogeneic bone marrow transplantation. Culprit medications in the fatal cases were sunitinib; phenytoin, or amoxicillin for the second fatal case; and carfilzomib, allopurinol, or 
Table 2 Culprit drugs responsible for SJS/TEN in adults, Clalit, 2008-19

\begin{tabular}{|c|c|c|c|c|c|c|c|}
\hline Drug name & $\begin{array}{l}\text { Number of } \\
\text { SJS/TEN } \\
\text { cases }\end{array}$ & $\begin{array}{l}\text { Number of SJS/ } \\
\text { TEN cases with true } \\
\text { diagnosis }\end{array}$ & $\begin{array}{l}\text { Number of SJS/TEN } \\
\text { cases with probable } \\
\text { diagnosis }\end{array}$ & $\begin{array}{l}\text { ALDEN } \\
\text { score } \\
\text { (mean) }\end{array}$ & SD & $\begin{array}{l}\text { Total adult new- } \\
\text { users of drug in the } \\
\text { study period }\end{array}$ & $\begin{array}{l}\text { SJS/TEN adult } \\
\text { cases/10,000 new } \\
\text { users of the drug }\end{array}$ \\
\hline Allopurinol & 11 & 10 & 1 & 5.18 & 1.85 & 100,141 & 1.10 \\
\hline Lamotrigine & 9 & 7 & 2 & 5.67 & 0.67 & 31,954 & 2.82 \\
\hline Phenytoin & 8 & 8 & 0 & 4.5 & 1.50 & 22,458 & 3.56 \\
\hline Amoxicillin & 6 & 6 & 0 & 3 & 1.53 & $2,190,927$ & 0.03 \\
\hline Ciprofloxacin & 6 & 2 & 4 & 3 & 1.00 & 846,757 & 0.07 \\
\hline Etoricoxib & 5 & 4 & 1 & 5.75 & 0.43 & 951,325 & 0.05 \\
\hline $\begin{array}{l}\text { Trimethoprim-sul- } \\
\text { famethoxazole }\end{array}$ & 4 & 4 & 0 & 2 & 2.92 & 153,670 & 0.26 \\
\hline Valproic acid & 4 & 2 & 2 & 3 & 0.82 & 58,729 & 0.68 \\
\hline Clindamycin & 4 & 3 & 1 & 2.5 & 0.87 & 120,435 & 0.33 \\
\hline Cefuroxime & 4 & 3 & 1 & 4 & 1.22 & $1,396,231$ & 0.02 \\
\hline $\begin{array}{l}\text { Amoxicillin-clavu- } \\
\text { lanate }\end{array}$ & 4 & 4 & 0 & 3.25 & 1.09 & $1,960,420$ & 0.02 \\
\hline Roxithromycin & 3 & 2 & 1 & 3 & 0.82 & 954,837 & 0.03 \\
\hline Etodolac & 3 & 3 & 0 & 4.33 & 0.47 & $1,356,200$ & 0.02 \\
\hline Dipyrone & 2 & 1 & 1 & 1.5 & 0.50 & $\mathrm{NA}^{\mathrm{a}}$ & NA \\
\hline Vancomycin & 2 & 2 & 0 & 1.5 & 1.50 & $\mathrm{NA}^{\mathrm{b}}$ & NA \\
\hline Bendamustine & 2 & 2 & 0 & 1 & 2.00 & 1660 & 12.05 \\
\hline Carbamazepine & 2 & 1 & 1 & 5 & 1.00 & 61,789 & 0.32 \\
\hline Ceftriaxone & 1 & 1 & 0 & 3 & NA & $\mathrm{NA}^{\mathrm{b}}$ & NA \\
\hline Acyclovir & 1 & 0 & 1 & 2 & NA & 242,284 & 0.04 \\
\hline Sulfasalazine & 1 & 1 & 0 & 6 & NA & 13,933 & 0.72 \\
\hline Terbinafine & 1 & 1 & 0 & 3 & NA & 400,982 & 0.02 \\
\hline Cefazolin & 1 & 1 & 0 & 2 or 4 & NA & $\mathrm{NA}^{\mathrm{b}}$ & NA \\
\hline Macrogol & 1 & 0 & 1 & 1 & NA & 266,939 & 0.04 \\
\hline Nitrofurantoin & 1 & 1 & 0 & 1 & NA & 351,227 & 0.03 \\
\hline Pralatrexate & 1 & 1 & 0 & 1 & NA & 26 & 384.62 \\
\hline Sunitinib & 1 & 1 & 0 & 4 & NA & 737 & 13.57 \\
\hline Ibuprofen & 1 & 1 & 0 & -2 & NA & $\mathrm{NA}^{\mathrm{a}}$ & NA \\
\hline Celecoxib & 1 & 1 & 0 & NA & NA & 273,590 & 0.04 \\
\hline Topiramate & 1 & 1 & 0 & 3 & NA & 18,901 & 0.53 \\
\hline Levomepromazine & 1 & 1 & 0 & 3 & NA & 11,490 & 0.87 \\
\hline Quetiapine & 1 & 1 & 0 & 1 & NA & 19,999 & 0.50 \\
\hline Citalopram & 1 & 1 & 0 & 0 & NA & 190,170 & 0.05 \\
\hline Hydroxychloroquine & 1 & 1 & 0 & 3 & NA & 27,336 & 0.37 \\
\hline Codeine & 1 & 1 & 0 & 1 & NA & 691,857 & 0.01 \\
\hline Ofloxacin & 1 & 1 & 0 & -2 & NA & 446,280 & 0.02 \\
\hline Carfilzomib & 1 & 0 & 1 & NA & NA & 452 & 22.12 \\
\hline Cabozantinib & 1 & 1 & 0 & 3 & NA & 35 & 285.71 \\
\hline Pregabalin & 1 & 1 & 0 & 0 & NA & 106,567 & 0.09 \\
\hline
\end{tabular}

NA not available, OTC over-the-counter, $S D$ standard deviation, SJS Stevens-Johnson syndrome, TEN toxic epidermal necrolysis

List of drugs by order of the total number of cases identified, along with mean ALDEN, and the frequency of occurrence of SJS/TEN per 10,000 adult new users of the drug. The list includes 2 optional culprit drugs for 14 patients and 3 optional culprit drugs for 2 patients

Culprit drug/s has not been identified during hospitalization, or discharge letters from hospitalization could not be reached in five of 87 valid cases, which are not represented in the table

ALDEN scores were calculated for 4 cases of etoricoxib and 3 cases of valproic acid, for which we had data available

${ }^{a}$ Data of OTC drugs dispensing are not available in the database. Thus, total numbers of new users of these drugs are missing

${ }^{\mathrm{b}}$ Data of inpatient treatments are not available in the searchable database. Thus, total numbers of new users of these drugs are missing 


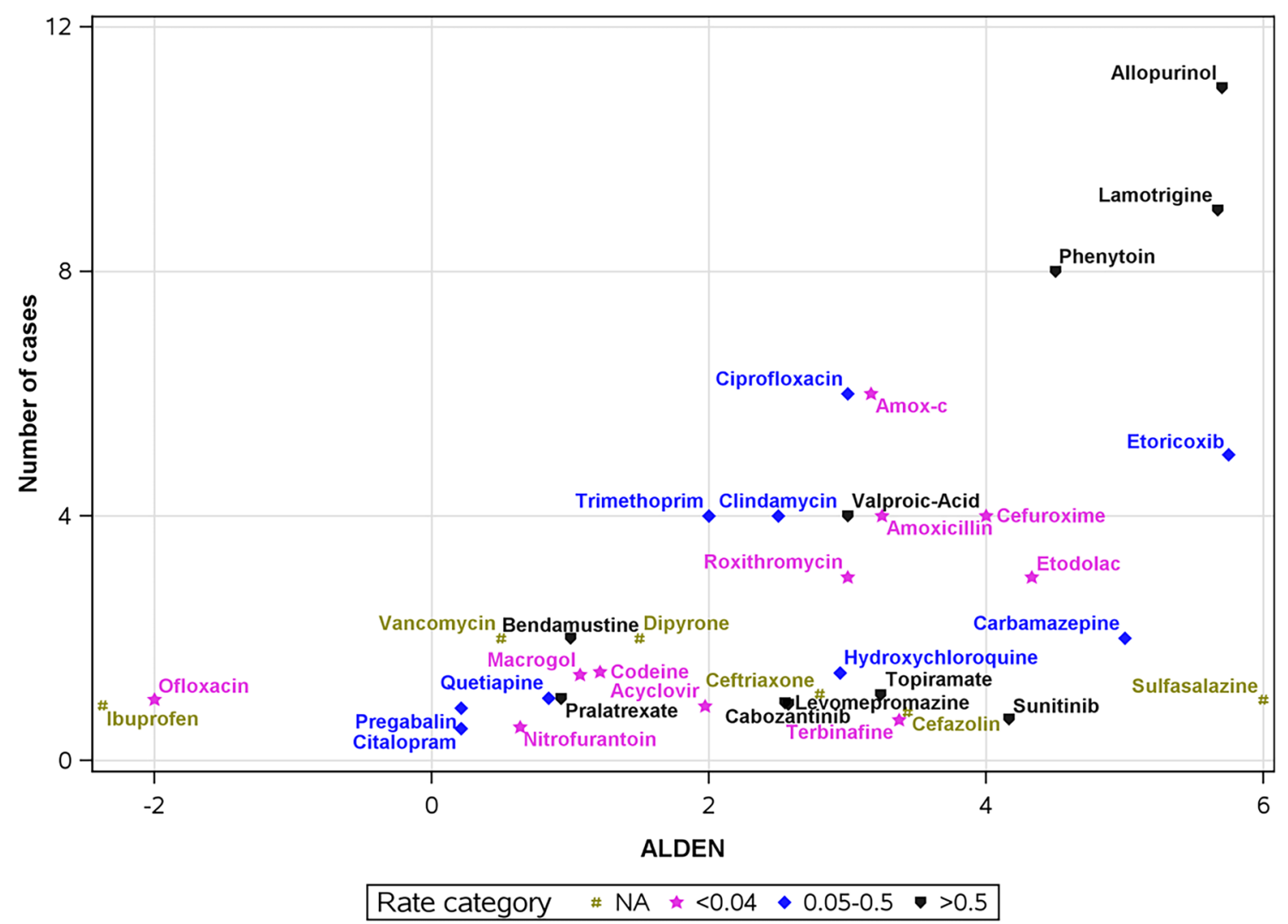

Fig. 1 Stevens-Johnson syndrome/toxic epidermal necrolysis culprit drugs identified in Clalit, 2008-19, presented by the mean ALDEN score on the $x$-axis and the number of cases associated with the drug between January 2008 and June 2019 on the $y$-axis. Occurrence rate/10,000 new users of the drug in the community is represented by drug symbols, divided into three tertiles and into a fourth category

trimethoprim-sulfamethoxazole for the third fatal case in which culprit drugs had been identified (see also Table S1 of the ESM).

\section{Discussion}

Stevens-Johnson syndrome and TEN are mucocutaneous adverse drug reactions that account for severe morbidity and high mortality. Culprit drug identification is crucial for clinical and regulatory purposes. Because of the rarity of the diseases, studies that were associating culprit drugs had been performed using spontaneous reporting systems [19], case series [20], or multinational studies that matched cases to control patients selected from hospitalized patients [4, 14]. In these case-control studies, the frequency of SJS/TEN occurrence within users of specific drugs might have been biased towards low estimates associated with drugs over-represented in hospitalized (control) patients relative to the general population. Frequency of drug use in the population was of unknown rate (NA) (when the total number of new users was unknown for over-the-counter and in-patient medications). Two drugs with unknown ALDEN scores, celecoxib and carfilzomib, are not represented in the figure. To prevent the overlapping of drug symbols in the figure, random jittering around the accurate location of points was applied, with a distance up to \pm 0.5

missing in these reports. Recently, Frey et al. have evaluated a population-based cohort from the UK Clinical Practice Research Datalink (CPRD) database, with cases diagnosed until 2013 [21,22]. Former seminal reports have discussed cases of SJS/TEN occurring 10-25 years ago [4, 11, 19, 20, 23]. New medications have been introduced into the market since then, and use of other drugs might have decreased. We aimed to describe the frequency of SJS/TEN in a large population-based cohort, provide an update of culprit drugs, and determine the frequency of occurrence of SJS/TEN for each drug. We used ALDEN scores to improve the assessment of drug causality in each case.

We found an incidence rate of 1.49-5.03 cases of SJS and TEN/million adults/year between 2008 and 2019. Incidence rate estimates from former reports were mostly around 1-2 per million persons (general population, including children) yearly [2].

In the study by Sassolas et al., drugs listed in decreasing order of their etiologic fraction, which was calculated from a case-control study using an ALDEN causality assessment, 
Table 3 Risks factors associated with SJS/TEN: univariate and multivariate analyses in a nested case-control study, Clalit, 2008-19

\begin{tabular}{|c|c|c|c|c|c|c|}
\hline Characteristic & $\begin{array}{l}\text { Adult SJS/TEN cases }(n \\
=87) \\
n(\%)\end{array}$ & $\begin{array}{l}\text { Controls }(n=1740) \\
n(\%)\end{array}$ & OR (95\% CI) & $p$ value & Adjusted $^{\mathrm{b}}$ OR $(95 \%$ CI) & $p$ value \\
\hline Age, mean, years (range) $)^{\mathrm{a}}$ & $56.96 \pm 19.7(20-88)$ & $56.94 \pm 19.6(20-89)$ & - & 0.990 & - & - \\
\hline Sex, female ${ }^{a}$ & $55(63.2)$ & $1100(63.2)$ & - & $>0.99$ & - & - \\
\hline Obesity (BMI > 30) & $34(39.1)$ & $437(25.1)$ & $2.01(1.26-3.19)$ & 0.003 & - & - \\
\hline Smoking (ever) & $33(37.9)$ & $581(33.4)$ & $1.25(0.78-1.99)$ & 0.359 & - & - \\
\hline Hyperlipidemia & $45(51.7)$ & $903(51.9)$ & $0.99(0.57-1.71)$ & 0.968 & - & - \\
\hline Hypertension & $38(43.7)$ & $626(36.0)$ & $1.74(0.97-3.12)$ & 0.062 & - & - \\
\hline Diabetes mellitus & $26(29.9)$ & $311(17.9)$ & $2.23(1.31-3.80)$ & 0.003 & $1.98(1.12-3.53)$ & 0.020 \\
\hline Malignancy & $26(29.9)$ & $208(12.0)$ & $3.69(2.18-6.27)$ & $<0.0001$ & $3.17(1.77-5.66)$ & $<0.0001$ \\
\hline Hypothyroidism & $15(17.2)$ & $168(9.7)$ & $2.03(1.12-3.71)$ & 0.021 & - & - \\
\hline $\begin{array}{l}\mathrm{S} / \mathrm{P} \text { cerebrovascular } \\
\text { accident }\end{array}$ & $15(17.2)$ & $102(5.9)$ & $3.82(2.01-7.27)$ & $<0.0001$ & $2.61(1.26-5.41)$ & 0.010 \\
\hline Ischemic heart disease & $14(16.1)$ & $228(13.1)$ & $1.35(0.70-2.61)$ & 0.378 & - & - \\
\hline Atrial fibrillation & $11(12.6)$ & $102(5.9)$ & $2.62(1.27-5.40)$ & 0.009 & - & - \\
\hline Epilepsy/convulsions & $8(9.2)$ & $24(1.4)$ & $7.17(3.13-16.42)$ & $<0.0001$ & $4.92(1.88-12.85)$ & 0.001 \\
\hline Psoriasis & $7(8.0)$ & $21(1.2)$ & $7.85(3.11-19.3)$ & $<0.0001$ & $10.28(3.61-29.31)$ & $<0.0001$ \\
\hline SLE & $2(2.3)$ & $1(0.1)$ & $40.0(3.60-441.3)$ & 0.003 & $17.41(1.31-230.72)$ & 0.030 \\
\hline $\begin{array}{l}\text { S/P bone marrow trans- } \\
\text { plantation }\end{array}$ & $2(2.3)$ & 0 & NA & 0.002 & NA & - \\
\hline $\begin{array}{l}\text { Previous allergy to any } \\
\text { medicine }\end{array}$ & $28(32.2)$ & $148(8.5)$ & $5.59(3.39-9.23)$ & $<0.0001$ & $5.21(2.95-9.19)$ & $<0.0001$ \\
\hline
\end{tabular}

$B M I$ body mass index, SJS Stevens-Johnson syndrome, TEN toxic epidermal necrolysis, NA not available, $O R$ odds ratio, SLE systemic lupus erythematosus, $S / P$ status post

${ }^{a}$ Matching variables

${ }^{b}$ Using multivariable models with a forward-selection algorithm; SLE, psoriasis, previous allergy to any medicine, epilepsy, malignancy, S/P CVA, and diabetes mellitus retained in the model

were allopurinol, carbamazepine, acetic acid non-steroidal anti-inflammatory drugs, nevirapine, sulfamethoxazole, phenytoin, macrolides, phenobarbital, cephalosporins, lamotrigine, other anti-infectious sulfonamides, quinolones, oxicam non-steroidal anti-inflammatory drugs, aminopenicillins, cyclines, and sertraline [11]. Allopurinol, lamotrigine, and phenytoin, with the largest number of SJS/TEN cases in the present study, confirmed this and other reports but in a different order of frequency [2, 11, 22]. We also confirm the high risk associated with COX-2 inhibitors, sulfonamides, cephalosporines, and carbamazepine, previously reported $[12,22]$. Clindamycin, amoxicillin, amoxicillin-clavulanate, ciprofloxacin, roxithromycin, ceftriaxone, and terbinafine, for which we found possible associations (ALDEN scores: 2-3) had been associated with case reports of SJS/TEN [23-30].

Sunitinib [31] and other small-molecule tyrosine-kinase inhibitors had been associated with SJS/TEN [32]. A possible association of SJS/TEN with the tyrosine kinase inhibitor cabozantinib is reported here for the first time. Interestingly, levomepromazine (named also methotrimeprazine)-associated SJS we describe had been reported only in two
Ashkenazi Jewish patients in Canada [33], thus providing a clue for a probable genetic factor that might play a role in risk. A case of TEN in a patient with COVID-19, which was assessed as probably related to hydroxychloroquine, has been recently published [34]. Finally, although we ascertained a low probability of association of bendamustine with SJS/TEN by the ALDEN score, there were only a few new users of the drug in Clalit; thus a high calculated rate of SJS/ TEN for 10,000 new users. Among cases in the FAERS, significant signals were previously associated with bendamustine [35]. Stevens-Johnson syndrome/toxic epidermal necrolysis cases associated with pralatrexate, carfilzomib, and quetiapine had not been previously reported. It will be important to follow up on these possible associations.

Antiepileptic drugs warrant special discussion. Frey et al. described absolute risks in a matched case-control study in which the exposure was defined by recorded prescription and association was ascertained by the ALDEN score, modified for a data-base analysis. Cumulative incidences reported by Frey et al. for phenytoin (4.59 cases/10,000 exposed) and lamotrigine (4.42 cases/10,000 exposed) were close to the cumulative incidence we report, and higher for 
carbamazepine (2.38 cases/10,000 exposed) [36]. These associations are highly reproducible and should serve in clinical decisions. We note possible associations with valproate and topiramate, while in Frey et al.'s cohort, although there were cases described in association with valproic acid (as well as with pregabalin), ALDEN suggested no causal association [36]; probable association was described by Sassolas et al. [11] and Roujeau et al. [14]. No observed cases of SJS/TEN among users of topiramate were observed by Frey et al. [36].

Association with other drugs mentioned as carrying possible risks for SJS/TEN such as corticosteroids [14] was not confirmed in the current cohort of 2.6-3 million adults over 11.5 years. Risk for nevirapine was also not confirmed in this cohort. We should note, however, that only 172 insurees were reported as using nevirapine at this time.

Systemic lupus erythematosus, human immunodeficiency virus, malignancies, and status post-bone marrow transplantation had been delineated in association with SJS/TEN [37]. T-cell dysfunction was suggested as a possible mechanism [5]. We confirmed risk associated with systemic lupus erythematosus and malignancy. Human immunodeficiency virus data are hidden to researchers in Clalit for privacy reasons, and could not be evaluated. One patient only, within our cases, was post-bone marrow transplantation, which did not permit a statistical analysis of risk. We have found a novel association with diabetes, where T-cell dysfunction had been elucidated $[38,39]$. We have detected for the first time a high risk associated with former allergies to drugs, and a risk associated with a previous diagnosis of psoriasis, which might warrant special attention. Risk associated with epilepsy and stroke is probably due to the co-occurrence of these diagnoses with the administration of antiepileptic drugs, and does not mean to highlight a cause-effect relationship. Nevertheless, these risk factors might serve to promote the identification of SJS/TEN in these patients earlier in the clinical course.

The average reported mortality had been 1-5\% in SJS and $25-35 \%$ in TEN [2]. We describe mortality of $6.9 \%$, which might reflect a higher proportion of SJS cases than TEN, in the present cohort, corresponding also to the low average SCORTEN score [1.89 (SD 1.23) in the entire cohort and 3.75 (SD 1.09) for the fatal cases]. In the SCORTENdevelopment cohort, mortality was $3.2 \%$ in patients with SCORTEN $0-1,12.1 \%$ for patients with SCORTEN 2, and close to 58\% with SCORTEN 3.75 [17].

This study has several limitations. The first limitation is the relatively low number of validated cases finally identified, and underestimation of the incidence rate of SJS/ TEN, and of cumulative incidence by each drug, that might have occurred because of a lack of available EHRs for six of the 173 potential cases, and because of potential underrecording of SJS/TEN, particularly in 2008-9, following introduction of the new specific ICD-9 codes for SJS/TEN. The second limitation is the lack of searchable data on overthe-counter drugs and inpatient treatments, which did not enable us to identify the total number of initiators to serve as the denominator in the calculation of cumulative incidence per number of initiators for these drugs. Third, it should be noted that one of the criteria used in the ALDEN score is notoriety of the drug, meaning that a higher score for the probability of being the culprit drug is given to a drug that had been ascertained as causing SJS/TEN before. This process might strengthen data on already-known culprit drugs, and might underestimate the probability of as yet unknown associations. Fourth, it is possible that underestimation of SCORTEN has occurred because of missing data we have replaced with zero scores. Finally, because our retrospective cohort study was observational in nature, it could not prove a cause-effect relationship regarding risk factors.

The strengths of this study include the collection of all cases occurring in a large unselected population during the last decade, from a database that contains full outpatient prescription data, allowing us to evaluate incident cases relative to the total number of new users. Other strengths include validation of all diagnoses, validation of the associated culprit drugs, as well as the calculation of ALDEN scores for all suspected drugs directly from the medical files.

\section{Conclusions}

Culprit drugs with ALDEN scores $\geq 4$ associated with the highest absolute risks of STS/TEN were phenytoin, lamotrigine, allopurinol, and sunitinib. Other drugs with ALDEN scores $\geq 4$ were sulfasalazine, carbamazepine, etoricoxib, etodolac, and cefuroxime. Risk factors associated with SJS/ TEN were psoriasis, former drug allergies, as well as a history of cerebrovascular accident and of diabetes mellitus, in addition to known associations with systemic lupus erythematosus and malignancies. These associations require confirmation in other studies.

Supplementary Information The online version contains supplementary material available at https://doi.org/10.1007/s40257-021-00661-0.

\section{Declarations}

Funding No funding was received for this work.

Conflict of interest No conflict of interest reported for all authors.

Ethics approval The Lady Davis Carmel Review Board approved the study protocol (0098-19-CMC).

Consent to participate Not applicable.

Consent for publication Not applicable. 
Availability of data and material Anonymized data will be available upon request by qualifying researchers.

Code availability Not applicable.

Author contributions DM, NS, and NG performed the analysis; DM, WS, and NG planned the study and interpreted the results; DM and NG drafted the manuscript.

\section{References}

1. Gerull R, Nelle M, Schaible T. Toxic epidermal necrolysis and Stevens-Johnson syndrome: a review. Crit Care Med. 2011;39(6):1521-32.

2. Harr T, French LE. Stevens-Johnson syndrome and toxic epidermal necrolysis. Chem Immunol Allergy. 2012;97:149-66.

3. Hsu DY, Brieva J, Silverberg NB, Silverberg JI. Morbidity and mortality of Stevens-Johnson syndrome and toxic epidermal necrolysis in United States adults. J Invest Dermatol. 2016;136:1387-97.

4. Mockenhaupt M, Viboud C, Dunant A, Naldi L, Halevy S, Bouwes Bavinck JN, et al. Stevens-Johnson syndrome and toxic epidermal necrolysis: assessment of medication risks with emphasis on recently marketed drugs. The EuroSCAR-study. J Invest Dermatol. 2008;128(1):35-44.

5. Bellón T. Mechanisms of severe cutaneous adverse reactions: recent advances. Drug Saf. 2019;42(8):973-92.

6. Torres MJ, Blanca M. The complex clinical picture of beta-lactam hypersensitivity: penicillins, cephalosporins, monobactams, carbapenems, and clavams. Med Clin North Am. 2010;94(4):805-20 (xii).

7. Chung WH, Hung SI, Chen YT. Human leukocyte antigens and drug hypersensitivity. Curr Opin Allergy Clin Immunol. 2007;7(4):317-23.

8. European Medicines Agency. Annex I: summary of product characteristics. Available from: https://www.ema.europa.eu/en/ documents/product-information/sildenafil-teva-epar-product-infor mation_en.pdf. Accessed 29 Nov 2021.

9. US FDA. CYMBALTA: highlights of prescribing information. Available from: https://www.accessdata.fda.gov/drugsatfda_docs/ label/2017/021427s049lbl.pdf. Accessed 29 Nov 2021.

10. DailyMed. GLIMEPIRIDE tablet. Available from: https://daily med.nlm.nih.gov/dailymed/lookup.cfm?setid=9b74fbac-9e044927-9981-1e3dace89c6d. Accessed 29 Nov 2021.

11. Sassolas B, Haddad C, Mockenhaupt M, Dunant A, Liss Y, Bork $\mathrm{K}$, et al. ALDEN, an algorithm for assessment of drug causality in Stevens-Johnson syndrome and toxic epidermal necrolysis: comparison with case-control analysis. Clin Pharmacol Ther. 2010;88(1):60-8.

12. Lonjou C, Borot N, Sekula P, Ledger N, Thomas L, Halevy S, et al. A European study of HLA-B in Stevens-Johnson syndrome and toxic epidermal necrolysis related to five high-risk drugs. Pharmacogenet Genomics. 2008;18:99-107.

13. Tassaneeyakul W, Jantararoungtong T, Chen P, Lin PY, Tiamkao $\mathrm{S}$, Khunarkornsiri U, et al. Strong association between HLAB*5801 and allopurinol-induced Stevens-Johnson syndrome and toxic epidermal necrolysis in a Thai population. Pharmacogenet Genomics. 2009;19(9):704-9.

14. Roujeau J, Kelly J, Naldi L, Rzany B, Stern R, Anderson T, et al. Medication use and the risk of Stevens-Johnson syndrome or toxic epidermal necrolysis. N Engl J Med. 1995;333(24):1600-7.

15. Reges O, Greenland P, Dicker D, Leibowitz M, Hoshen M, Gofer I, et al. Association of bariatric surgery using laparoscopic banding, Roux-en-Y gastric bypass, or laparoscopic sleeve gastrectomy vs usual care obesity management with all-cause mortality. JAMA. 2018;319(3):279-90.

16. Dagan N, Barda N, Kepten E, Miron O, Perchik S, Katz MA, et al. BNT162b2 mRNA Covid-19 vaccine in a nationwide mass vaccination setting. N Engl J Med. 2021. https://doi.org/10.1056/ NEJMoa2101765 (Epub ahead of print).

17. Bastuji-Garin S, Fouchard N, Bertocchi M, Roujeau JC, Revuz J, Wokenstein P. SCORTEN: a severity of illness score for toxic epidermal necrolysis. J Invest Dermatol. 2000;115:149-53.

18. The RegiSCAR Project. Available from: http://www.regiscar.org/ Office_1.html. Accessed 29 Nov 2021.

19. Abe J, Mataki K, Umetsu R, Ueda N, Kato Y, Nakayama Y, Kinosada Y, et al. Stevens-Johnson syndrome and toxic epidermal necrolysis: the Food and Drug Administration adverse event reporting system, 2004-2013. Allergol Int. 2015;64(3):277-9.

20. Guillaume JC, Roujeau JC, Revuz J, Penso D, Touraine R. The culprit drugs in 87 cases of toxic epidermal necrolysis (Lyell's syndrome). Arch Dermatol. 1987;123(9):1166-70.

21. Frey N, Bircher A, Bodmer M, Jick SS, Meier CR, Spoendlin J. Antibiotic drug use and the risk of Stevens-Johnson syndrome and toxic epidermal necrolysis: a population-based case-control study. J Invest Dermatol. 2018;138(5):1207-9.

22. Frey N, Bircher A, Bodmer M, Jick SS, Meier CR, Spoendlin J. Validation of Stevens-Johnson syndrome or toxic epidermal necrolysis diagnoses in the Clinical Practice Research Datalink. Pharmacoepidemiol Drug Saf. 2017;26(4):429-36. https://doi.org/ 10.1002/pds.4124 (Epub 2016 Nov 20).

23. Sousa-Pinto B, Araújo L, Freitas A, Correia O, Delgado L. Stevens-Johnson syndrome/toxic epidermal necrolysis and erythema multiforme drug-related hospitalisations in a national administrative database. Clin Transl Allergy. 2018;22(8):2.

24. Sahagún Flores JE, Soto Ortiz JA, Tovar Méndez CE, Cárdenas Ochoa EC, Hernández FG. Síndrome de Stevens Johnson más colestasis intrahepática inducido por clindamicina o clorfeniramina [Stevens-Johnson syndrome plus intrahepatic cholestasis caused by clindamycin or chlorpheniramine]. Dermatol Online J. 2009;15(5):12 (Spanish).

25. Rodríguez-Martín S, Martín-Merino E, Lerma V, RodríguezMiguel A, González O, González-Herrada C, et al. Active surveillance of severe cutaneous adverse reactions: a case-population approach using a registry and a health care database. Pharmacoepidemiol Drug Saf. 2018;27(9):1042-50.

26. Limauro DL, Chan-Tompkins NH, Carter RW, Brodmerkel GJ Jr, Agrawal RM. Amoxicillin/clavulanate-associated hepatic failure with progression to Stevens-Johnson syndrome. Ann Pharmacother. 1999;33(5):560-4.

27. Okan G, Yaylaci S, Peker O, Kaymakoglu S, Saruc M. Vanishing bile duct and Stevens-Johnson syndrome associated with ciprofloxacin treated with tacrolimus. World J Gastroenterol. 2008;14(29):4697-700.

28. Pejčić AV. Stevens-Johnson syndrome and toxic epidermal necrolysis associated with the use of macrolide antibiotics: a review of published cases. Int J Dermatol. 2021;60(1):12-24.

29. Narayanan VS, Mamatha GP, Ashok L, Rajashekar N. Steven Johnson syndrome due to I.V ceftriaxone: a case report. Indian J Dent Res. 2003;14(4):220-3.

30. Banik B, Bhar D, Sil A. Terbinafine-induced Steven-Johnson syndrome and toxic epidermal necrolysis (SJS/TEN) overlap. Postgrad Med J. 2021;97(1152):630-31.

31. Lee JH, Lee JH, Lee JH, Kim SY, Kim GM. Case of sunitinib-induced Stevens-Johnson syndrome. J Dermatol. 2013;40(9):753-4.

32. $\mathrm{Ng} \mathrm{CY}$, Chen CB, Wu MY, Wu J, Yang CH, Hui RC, et al. Anticancer drugs induced severe adverse cutaneous drug reactions: an updated review on the risks associated with 
anticancer targeted therapy or immunotherapies. J Immunol Res. 2018;17(2018):5376476.

33. Moubayed D, Gifuni AJ, Tourian L. Methotrimeprazine-associated Stevens-Johnson syndrome in 2 Ashkenazi Jewish patients. J Clin Psychopharmacol. 2017;37(1):112-3.

34. Rossi CM, Beretta FN, Traverso G, Mancarella S, Zenoni D. A case report of toxic epidermal necrolysis (TEN) in a patient with COVID-19 treated with hydroxychloroquine: are these two partners in crime? Clin Mol Allergy. 2020;6(18):19.

35. Rosen AC, Balagula Y, Raisch DW, Garg V, Nardone B, Larsen N, et al. Life-threatening dermatologic adverse events in oncology. Anticancer Drugs. 2014;25(2):225-34.

36. Frey N, Bodmer M, Bircher A, Rüegg S, Jick SS, Meier CR, et al. The risk of Stevens-Johnson syndrome and toxic epidermal necrolysis in new users of antiepileptic drugs. Epilepsia. 2017;58(12):2178-85.
37. Mandelcorn R, Shear NH. Lupus-associated toxic epidermal necrolysis: a novel manifestation of lupus? J Am Acad Dermatol. 2003;48:525-9.

38. Nyambuya TM, Dludla PV, Mxinwa V, Nkambule BB. A systematic review and meta-analysis on the regulation of programmed cell death-1 on T-cells in type 2 diabetes. Medicine (Baltimore). 2021;100(15): e25488. https://doi.org/10.1097/MD.0000000000 025488.

39. Xia C, Rao X, Zhong J. Role of T lymphocytes in type 2 diabetes and diabetes-associated Inflammation. J Diabetes Res. 2017;2017:6494795. https://doi.org/10.1155/2017/6494795 (Epub 2017 Jan 31). 